\title{
Structure and spectra of photochemically obtained nanosized silver particles in presence of modified porous silica
}

\author{
Galina Krylova, ${ }^{1}$ Anna Eremenko, ${ }^{1, \dagger}$ Natalia Smirnova, ${ }^{1}$ and Susie Eustis ${ }^{2}$ \\ ${ }^{1}$ Institute of Surface Chemistry, Ukrainian National Academy of Sciences, \\ 17 General Naumov str., Kyiv 03164, Ukraine \\ ${ }^{2}$ Laser Dynamic Laboratory, School of Chemistry and Biochemistry, \\ Georgia Institute of Technology, Atlanta, GA 30332, USA
}

\begin{abstract}
Mesoporous silica powders and films modified with organic sensitizer benzophenone were used as photocatalysts in the reaction of silver ion reduction by isopropyl alcohol under UV-irradiation with $\lambda=253.7 \mathrm{~nm}$ and $365 \mathrm{~nm}$ in presence of colloidal silica as stabilizer. Morphological changes of silver colloids during irradiation were studied using transmission electron microscopy, and correlated to the absorption spectra.
\end{abstract}

\section{INTRODUCTION}

The preparation of small metal particles at the nano scale has received increasing attention due to unique properties of such particles which result from sizeeffect [1, 2]. The most significant is that silver nanoparticles have a strong surface plasmon resonance (SPR) absorption band in the visible region which cause these samples to display brilliant colors [3]. In this context a number of methods have been applied to generate silver nanoparticles [4-7]. The photochemical methods have several advantages e.g. easy control of reaction kinetics $[7,8]$.

It is known, that fine silver particles could be prepared by photolysis of $\mathrm{AgNO}_{3}$ solutions in presence of isopropil alcohol with UV light $(\lambda=253.7 \mathrm{~nm})$ [9]. The purpose is to sensitize the photoreduction reaction to visible or near UV light. Benzophenone (BP) has received the most attention among of all photochemical sensitizers due to the ability of the excited triplet state to abstract $\mathrm{H}$ atoms from aliphatic alcohols (under $\lambda=355-365 \mathrm{~nm}$ irradiation) with the formation of ketyl radicals [10] which are well known as effective photoreducing agents [11]. However BP solubility in water is negligible.

Thus in this work we propose modification of the silica surface with BP molecules via physical adsorption, which generates heterogeneous photosensitive materials, perspective as redox photocatalysts. The photonics of BP adsorbed on silica is similar to that in liquid systems [12]. Recently we studied growth and fragmentation of silver nanoparticles in their synthesis with laser and UV light by photo-sensitization with benzophenone [13]. The main goal of this work is to study photochemical formation of nanosized silver colloids

\footnotetext{
†E-mail: annaerem@voliacable.com
}

in presence of mesoporous silica modified with (BP), as photocatalyst.

\section{EXPERIMENTAL}

2.1. Reagents. The following chemicals are used: Silicagel (SG-150Å Davisil) with specific surface area of $300 \mathrm{~m}^{2} / \mathrm{g}$ (Aldrich), colloidal silica Du Pont Ludox AM-30 with surface area 210-230 M² / (Aldrich), $\mathrm{AgNO}_{3}$, sodium dodecylsulphate (SDS, Aldrich), and tetraethoxysilane 98\% (TEOS, Aldrich) were used as received. Benzophenone (BP, Fluka) was purified by double recrystallization from ethanol. Isopropyl alcohol (Fluka) and hexane (Fluka) were purified by repeated distillation.

2.2. Film preparation. The mesoporous silica films have been prepared by the sol-gel method using two kinds of templates: cationic surfactant cetyltrimethylammonium bromide (CTAB, Aldrich) and nonionic tri-block copolymer Pluronic $\mathrm{EO}_{20} \mathrm{PO}_{70} \mathrm{EO}_{20}$ (P123, Aldrich) by the method described in literature [14-16]. The precursor sol was prepared by hydrolysis of TEOS in a mixture of distilled water, ethanol (Fluka), and $1 \mathrm{M}$ $\mathrm{HNO}_{3}$ solution. Twenty-four hours after hydrolysis, either an ethanolic solution of P123 or a water solution of $\mathrm{CTAB}$ was added to this solution. The total molar ratios were 1 TEOS: 0.1 CTAB: $0.02 \mathrm{HNO}_{3}$ : $10 \mathrm{H}_{2} \mathrm{O}: 5$ $\mathrm{C}_{2} \mathrm{H}_{5} \mathrm{OH}$ and 1 TEOS: 0.008 P123: $0.16 \mathrm{HNO}_{3}$ : $15 \mathrm{H}_{2} \mathrm{O}$ : $15 \mathrm{C}_{2} \mathrm{H}_{5} \mathrm{OH}$. To prepare $\mathrm{Ag}^{+}$-containing $\mathrm{SiO}_{2}$ coatings with $0.1 \mathrm{Ag} / \mathrm{SiO}_{2}$ atomic ratios, aqueous solutions of $\mathrm{AgNO}_{3}$ were added to the hydrolyzed silica sol before film deposition procedure.

The coatings were deposited onto a clean glass substrates by the dip-coating technique. The films were dried for 12 hours at ambient temperature, followed 
by heat treatment at $400^{\circ} \mathrm{C}$ with a rate of $1^{\circ} \mathrm{C} / \mathrm{min}$ and calcined at $400{ }^{\circ} \mathrm{C}$ for $6 \mathrm{~h}$.

The adsorption of $\mathrm{BP}$ on silicagel (SG) and $\mathrm{SiO}_{2}$ films was carried out from hexane solution. To measure the adsorption isotherm, the samples were thermoactivated at $250^{\circ} \mathrm{C}$, and silica was placed in the BP hexane solution with the initial concentration in the range $10^{-5}$ to $10^{-3} \mathrm{M}$ for $24 \mathrm{~h}$ to achieve the adsorption equilibrium. The amount of adsorbed BP molecules was monitored by the measurements of the optical density of BP at $355 \mathrm{~nm}$ using Lambda UV-Vis spectrophotometer (Perkin Elmer). We detected negligible BP desorption from silica-BP system after prolonged contact of silica$\mathrm{BP}$ with water under stirring.

2.3. Photocatalytic experiment. A monochromatic 1000-W low-pressure mercury lamp was used as an irradiation source for $253.7 \mathrm{~nm}$ light. A 500-W highpressure mercury lamp combined with the appropriate UV 7-60 filter was used as a source of $365 \mathrm{~nm}$ light. The reaction cell was a cylindrical quartz vessel having a volume of $25 \mathrm{ml}$. Incident photon intensity as determined by a tris(oxalato)ferrate(III)actinometer [17] was determined at $253.7 \mathrm{~nm}$ and $365 \mathrm{~nm}$ to be $2 * 10^{17}$ and $2.12 * 10^{15} \mathrm{~cm}^{-2} \mathrm{~s}^{-1}$, respectively. Irradiation of the solution was carried out while bubbling argon through the solution at room temperature. The absorption spectra of the solutions before and after irradiation were measured with a Lambda UV-Vis spectrophotometer (Perkin Elmer) in a rectangular $1 \mathrm{~cm}$ thick quartz cell. Diffusion reflectance spectra of SG-BP powders were also measured with this spectrophotometer.

Porous silica powders and $\mathrm{SiO}_{2}$ films with adsorbed BP have been investigated as catalysts in the photochemical reduction of silver ions in water-alcohol solvent under mercury lamp irradiation. In accordance with Yonezawa et al. [9], colloid silica plays role of stabilizer of colloid nanoparticles in solution and prevents their precipitation and aggregation. An aqueous solution containing $\mathrm{AgNO}_{3}\left(1.5 * 10^{-4} \mathrm{M}\right)$, i-pro $(0.4 \mathrm{M})$, and Ludox (1\%) was irradiated with $254 \mathrm{~nm}$ or $365 \mathrm{~nm}$ light in presence of $\mathrm{SiO}_{2}$ with adsorbed BP (films or powders). The catalyst is removed immediately after irradiation and the absorption spectra of the irradiated solutions are measured.

2.4. Structural investigations. X-ray diffraction (XDR, $\mathrm{Cu} \mathrm{K}_{\alpha}$ radiation) analysis was performed on the thin films using a diffractometer DRON-3 with the minimum glancing angle $\theta=0.5$. Hexane vapor adsorption isotherms on the porous $\mathrm{SiO}_{2}$ films were recorded on vacuum adsorption apparatus. Specific surface area ( $\left.\mathrm{S}_{\mathrm{BET}}\right)$ was established using adsorption data in the range of 0.04 to 0.2 . Pore size distribution was calculated by desorption branch according Kelvin equation [18].

For the TEM measurements, a drop of solution was allowed to dry overnight on a carbon coated copper
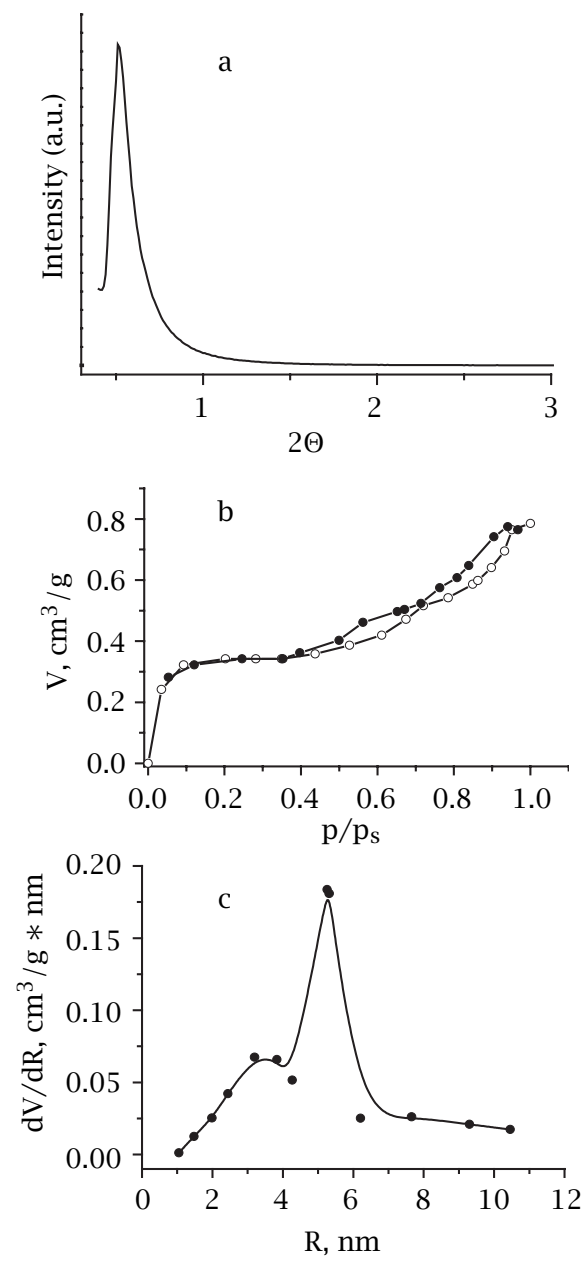

Figure 1. (a) XRD patterns of $\mathrm{SiO}_{2}$ film synthesized using P123 as a template; (b) hexane vapor adsorption -o- and desorption -•- isotherms and (c) pore size distribution in this film.

TEM grid (Ted Pella). The silver nanoparticle solutions were imaged using an accelerating voltage of $100 \mathrm{kV}$ in the JOEL JEM-100C transmission electron microscope (TEM).

\section{RESULTS AND DISCUSSION}

Mesoporous silica films and powders act as carriers for the photoactive molecule BP, possess highly developed surface area with large adsorption capacity to the BP molecules and are stable in various reaction media. Use of templates such as nonionic triblock copolymer P123 resulted in the formation of regular hexagonal structure of the film. XRD images and characteristics of the porosity obtained via the hexane vapor adsorption measurements for the synthesized samples are shown at Figure 1. It is seen that $\mathrm{SiO}_{2}$ film prepared in presence of the template agent $\mathrm{P} 123$ possesses bimodal pore size 


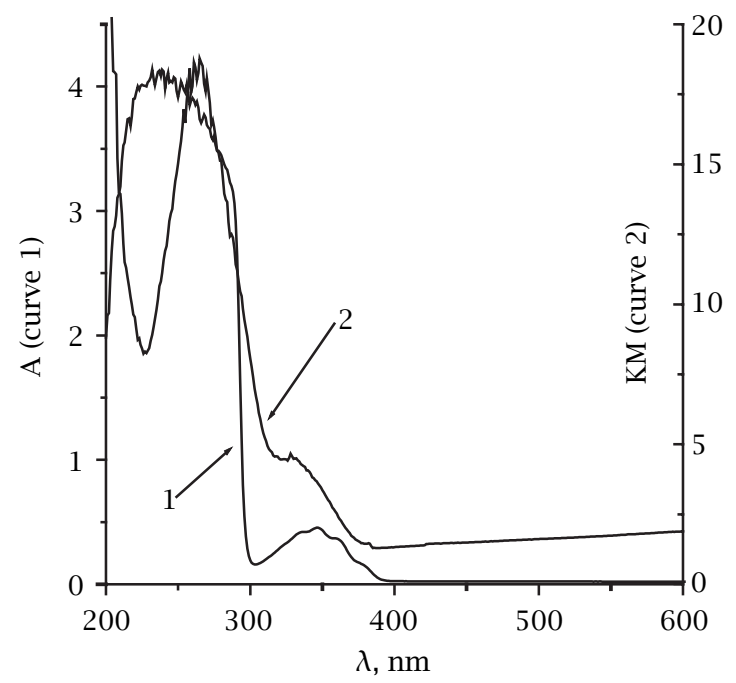

Figure 2. (1) Absorption spectrum of $4.3 \times 10^{-3} \mathrm{M}$ hexane solution of $B P$. (2) Diffusion reflection spectrum of $S G-B P_{\mathrm{ads}}$ powder $\left(\Gamma_{B P}=1.42 \times 10^{-4} \mathrm{~mol} / \mathrm{g}\right)$ transformed by ShusterKubelka-Munk equation.

distribution with radius of the smaller ones' 3.3 and of the bigger ones' $5.7 \mathrm{~nm}$. From XRD pattern one could obtain the value of unit cell parameter $\mathrm{a}=18.56 \mathrm{~nm}$ (assuming that the pore structure is hexagonal). So the thickness of the walls has been easily estimated to be about $7.2 \mathrm{~nm}$. Specific surface area of the film has been determined from BET adsorption data analysis and equals to $\mathrm{S}_{\mathrm{BET}}=658 \mathrm{~m}^{2} / \mathrm{g}$. Such structures with large pores and thick walls are very stable for thermal and hydrothermal treatment and might be used in the large number of surface modification and photocatalitic reduction cycles.

Adsorption-desorption isotherm of BP from hexane solution on SG has Langmuir type and demonstrates the physical character of adsorption. The desorption curve demonstrates BP molecules are strongly bonded with silica surface (about $10 \%$ of the monolayer content). On Figure 2 it is seen that the diffusion reflection spectrum of BP adsorbed on silicagel is blue shifted in comparison to the spectrum of BP in solution $\left(\lambda_{\max }=346 \mathrm{~nm}\right)$, it gives the evidence that $\mathrm{C}=\mathrm{O}$ groups of $\mathrm{BP}$ molecules interact with $\mathrm{OH}$-groups of silica surface with formation of strong hydrogen bonds [19]. The absorption bands of BP-260 nm corresponds to $\pi \pi^{*}$ transition, and $346 \mathrm{~nm}$ corresponds to the forbidden $n \pi^{*}$ transition. Under excitation BP converts to the biradical triplet state, in which it abstracts hydrogen atom from aliphatic alcohols with the formation of two ketyl-radicals. They undergo protolytic dissociation, and the radical anion, which has a very high negative electrochemical potential, may be the true reductant $[7,20,21]$.

Porous silica powders and $\mathrm{SiO}_{2}$ films with adsorbed BP have been investigated in the photochemical reac- tion of silver ions reduction in water-alcohol solution under mercury lamp irradiation. The aqueous solution contained $\mathrm{AgNO}_{3}$, IPA and Ludox. It was irradiated in presence of $\mathrm{SiO}_{2}-\mathrm{BP}_{\text {ads }}$ (films or powders) with $254 \mathrm{~nm}$ or $365 \mathrm{~nm}$ light. The absorption spectra of reduced silver particles obtained under $254 \mathrm{~nm}$ irradiation in presence of silica-BP film are shown in Figure 3. It is seen that a broad absorption band of nanosized colloidal silver particles with the maximum at $407 \mathrm{~nm}$ appeared. When the silicagel- $\mathrm{BP}_{\mathrm{ads}}$ powder (with the weight of silica and silica/BP ratio equal to the ones on the film) was used as a photocatalyst, the colloidal silver particles accumulation occurred slower, perhaps due to the light scattering in water-SG dispersion.

The light absorption by small metal particles is described by Mie's theory [22]. According to this theory, when the particles are spherical and small in comparison with the wavelength of light (between 3 to $20 \mathrm{~nm}$ ), there is no strong dependence of the absorption spectra on particle size. This is because the particle sizes are below the size where higher-order terms in Mie's theory for the absorption coefficient become significant. Thus, only the dipole term, which depends on the total metal concentration in the solution, and not on the particle size has to be regarded. So the intensity of absorption band at $\lambda=407 \mathrm{~nm}$ is proportional to the nanoparticles concentration, and is plotted versus irradiation time in Figure 4 . Under irradiation with $\lambda=254 \mathrm{~nm}$, in the presence of $\mathrm{SiO}_{2}$-BP film the photoreduction process is more effective than without the film (curve 2). Under irradiation with $365 \mathrm{~nm}$ light the reaction rate of photoreduction decreased almost one order, that is in agreement with the decreasing light intensity (see experimental). Under $365 \mathrm{~nm}$ irradiation, when the silica film is absent, the silver nanoparticles formation is not observed. So it can be inferred, that under far UV-irradiation $\mathrm{SiO}_{2}-\mathrm{BP}$ systems act like the catalysts, making the reaction go faster, and they sensitize the process to the near UVlight, acting like photosensitizers.

The dependence of photoreduction kinetics on the $\mathrm{pH}$ values of the reaction solution shows that at the acidic condition reduction proceeds slower, and equilibrium does not achieve a saturation value. The reduction proceeds faster under neutral and basic conditions [23]. This confirms the suggestion that anion-radical, but not ketyl-radical itself is an active species in silver ion photoreduction, due to the fact that in acidic solution the protolytic dissociation with the formation of BP anion-radical is depressed [24]:

$$
\left(\mathrm{C}_{6} \mathrm{H}_{5}\right)_{2} \mathrm{C}^{\cdot} \mathrm{OH} \Longleftrightarrow\left(\mathrm{C}_{6} \mathrm{H}_{5}\right)_{2} \mathrm{C}^{\cdot} \mathrm{O}^{-}+\mathrm{H}^{+}
$$

The proposed reaction mechanism includes formation of triplet state of adsorbed BP followed by hydrogen atom abstraction by BP triplet from i-propanol and formation of two ketyl radicals, then protolytic dissociation of ketyl radicals and reduction of silver 


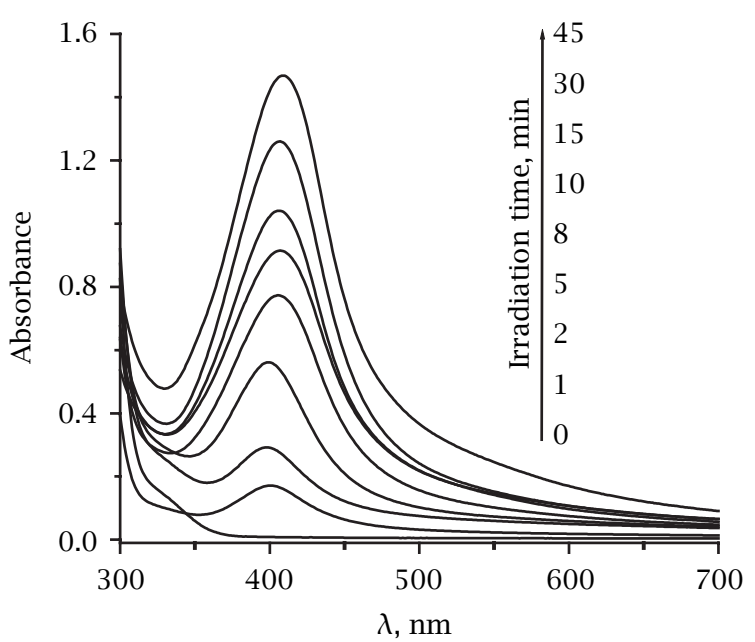

Figure 3. Absorption spectra of Ag nanoparticles accumulated in solution under irradiation in presence of $\mathrm{SiO}_{2}-B P_{\text {ads }}$ films.

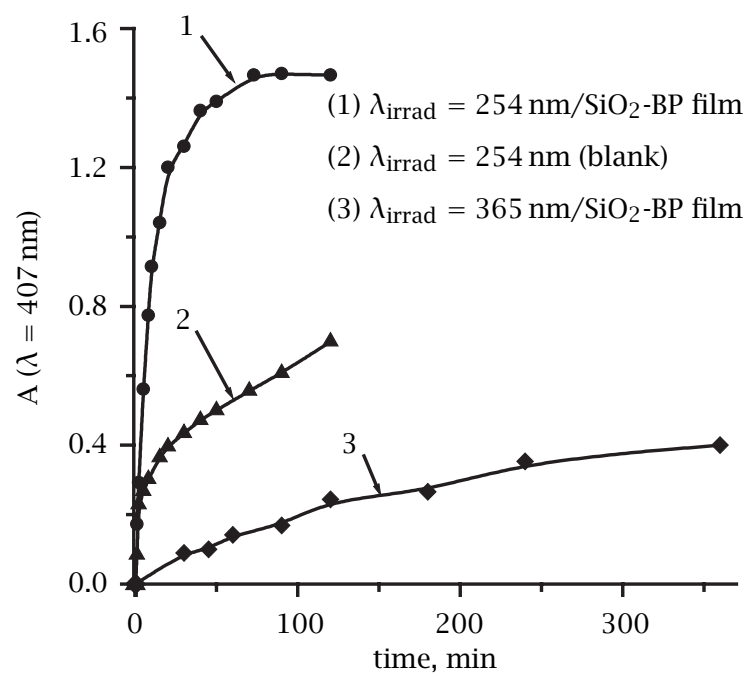

Figure 4. Kinetics of Ag nanoparticle formation during irradiation of $\mathrm{AgNO}_{3}$ solution in presence of $\mathrm{SiO}_{2}-B P$ films.

ions by the anion radicals leading to silver nanoparticle formation.

Mie's theory for the calculation of absorption coefficient of small metal particles in solution looks as follows:

$$
\alpha=\frac{18 \pi}{\ln 10} \frac{10^{5}}{\lambda} \frac{M n_{0}^{3}}{\rho}\left(\frac{\varepsilon_{2}}{\left(\varepsilon_{1}+2 n_{0}^{2}\right)+\varepsilon_{2}^{2}}\right)
$$

where $\alpha$ is the absorption coefficient (in $\mathrm{mol}^{-1} \cdot \mathrm{L} \cdot \mathrm{cm}^{-1}$ ), $\lambda$ is the wavelength of light (in $\mathrm{nm}), M$ and $\rho$ are the molecular weight and density of the metal, respectively, $n_{0}$ is the refractive index of the solvent and $\varepsilon_{1}, \varepsilon_{2}$ are the real and imaginary parts of the dielectric constant of the metal. When the size of

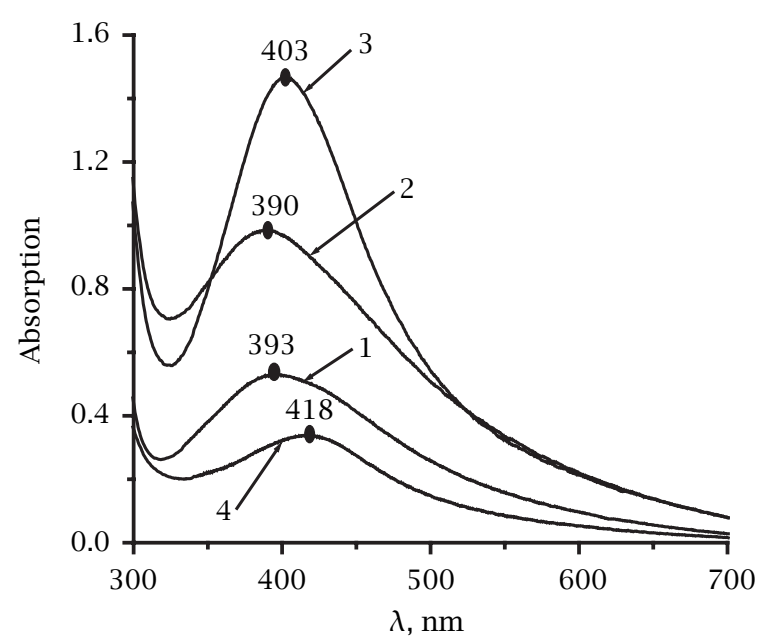

Figure 5. Absorption spectra of Ag particles in the solution in presence of Ludox after photolysis with $\mathrm{SiO}_{2}-B P$ film for (1) $1 \mathrm{~min}$, (2) $20 \mathrm{~min}$, (3) $45 \mathrm{~min}$, (4) $120 \mathrm{~min}$.

the particles becomes smaller than the mean free path of the electrons ( $52 \mathrm{~nm}$ for silver), the size-corrected value of $\varepsilon_{2}$ is to be used:

$$
\varepsilon_{2}^{\prime}=\varepsilon_{2}+\left(\omega_{p}^{2} / \omega^{3}\right) *\left(v_{F} / R\right)
$$

where $\omega$ is the light frequency, $\omega_{p}$ the plasmon frequency $\left(1.4 \times 10^{-16} \mathrm{~s}^{-1}\right.$ for silver $), v$ the electron velocity on the Fermi level, and $R$ the particle radius [25]. These two equations let us evaluate absorbance intensities, knowing silver particle's radius and vice versa.

Changes in the absorption spectrum of colloidal solution during Ag nanoparticle formation (after photolysis in presence of $\mathrm{SiO}_{2}$-BP film) are presented in Figure 5. Corresponding changes in particles' size distribution and morphological changes monitored by TEM are shown in Figures 6 and 7.

After 1 min of irradiation a lot of small particles (with $3 \mathrm{~nm}$ diameter) have been formed. Particles with the small radius possess wide and low intensity SPR bands (see Figure 5 curve 1), which is in agreement with eqs. (1), (2), and the expression for bandwidth at halfmaximum absorption:

$$
w=\frac{\left(\varepsilon_{0}+2 n_{0}^{2}\right) c m_{e} u_{F}}{2 N_{e} e^{2} R}
$$

where $m_{e}$ and $e$ are the electron' mass and charge, respectively, $\varepsilon_{0}$ is the high-frequency dielectric constant of the metal, and $N_{e}$ is the density of free electrons on the metal nanoparticles.

After 20 min of irradiation the small particles agglomerate and form large particles (Figure 6, curve 2) with a sharp intense absorption band. 


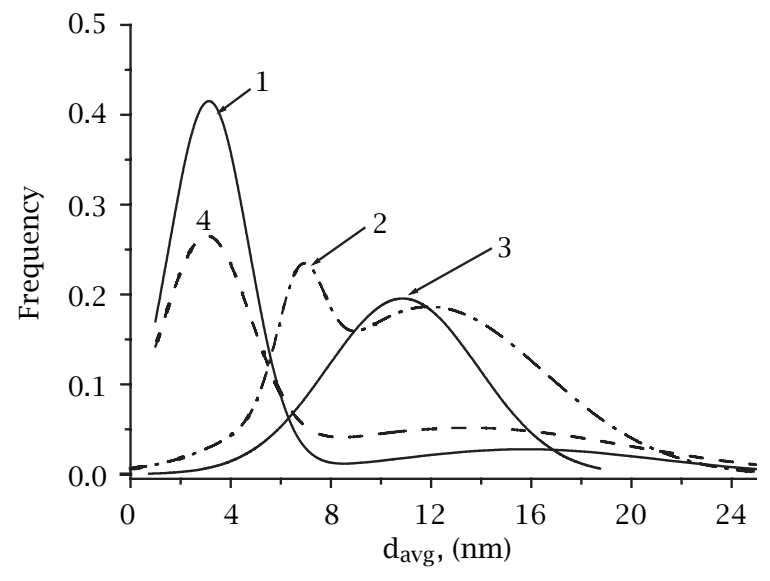

Figure 6. Size distribution of Ag nanoparticles (Gauss fitting) in colloids obtained in presence of Ludox after photolysis with $\mathrm{SiO}_{2}-B P$ film for (1) $1 \mathrm{~min}$ (2) $20 \mathrm{~min}$, (3) $45 \mathrm{~min}$, (4) $120 \mathrm{~min}$.

After 45 min of irradiation, when photoreduction process is almost finished, the particles are large and adsorbed on the surface of silica spheres of Ludox. Adsorption takes place perhaps due to the fact that positively charged colloidal silica surface (stabilized by quaternary amines) attract negatively charged Ag nanoparticles. Absorption spectrum becomes more intensive due to continuing formation of nanoparticles and red shifted due to adsorption, which causes some changes in dielectric constant of the Ag particles' surrounding.

After 2 hours of irradiation and 2 weeks of aging, the intensity of Ag colloid solution absorption spectrum is sharply decreased, perhaps due to sedimentation of large Ag particles and their aggregates with silica. Only a few fine Ag nanoparticles are left in the solution (Figure 7 image 4).

Based on eqs. (1) and (2) the molar absorption coefficient of colloidal Ag nanoparticles at the stage when the equilibrium in photoreduction reaction has been achieved (practically all $\mathrm{Ag}^{+}$ions are reduced, which is verified by the qualitative reaction with dithizone [26] after 45 minutes of irradiation), is calculated to be $\alpha=1.7 \times 10^{4} \mathrm{M}^{-1} \mathrm{~cm}^{-1}$ per mole of $\mathrm{Ag}$ atoms (with the consideration of size distribution on Figure 6 , curve 3 ). The experimental value of $\alpha=1.46 \times 10^{4}$ is simply evaluated from the maximum absorption at around $407 \mathrm{~nm}$ (Figure 3). The difference may be due to adsorption of silver nanoparticles on colloidal silica and formation of large Ag agglomerates.

The position of SPR band maximum of Ag colloid is red-shifted as compared to the maximum in pure water at $390 \mathrm{~nm}$ [3], especially big deviation is observed for spectrum 4 (Figure 5). In the accordance with Mie's theory the position of SPR band maximum for certain metal depends on dielectric properties of the solvent
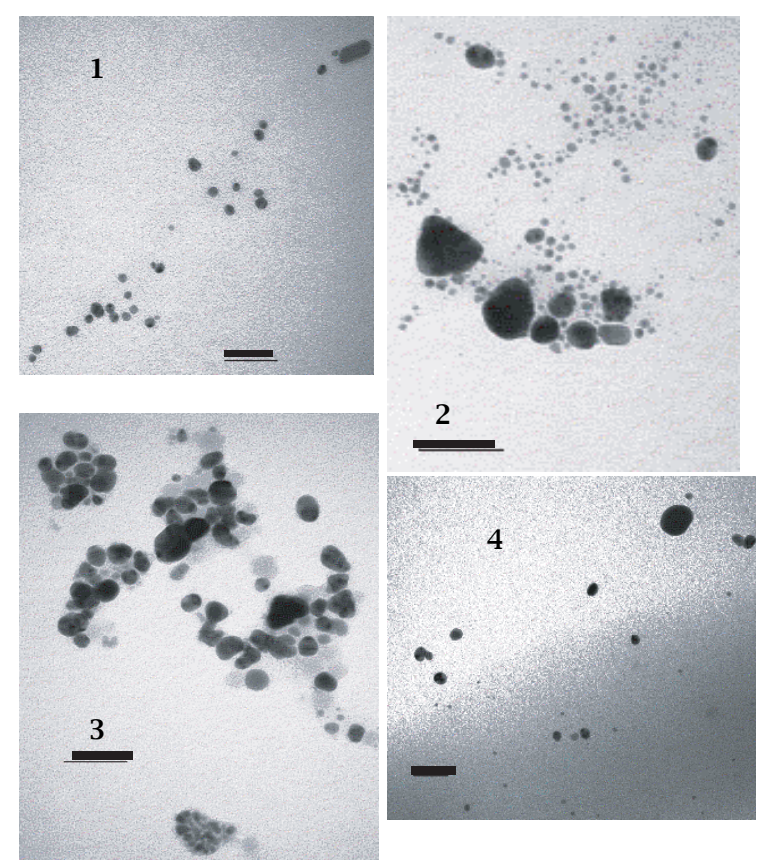

Figure 7. TEM images of Ag nanoparticles of samples shown in Figures 5 and 6 (with the same numeration). Scale bar on the images corresponds to $50 \mathrm{~nm}$.

and free electron density:

$$
\lambda_{\max }^{2}=\frac{(2 \pi c)^{2} m_{e}\left(\varepsilon_{0}+2 n_{0}^{2}\right)}{4 \pi e^{2} N_{e}}
$$

The measured value of refractive index of colloidal solution $n_{0}=1.34$ slightly differs from the refractive index of water $\left(n_{0}=1.333\right)$. Such changes may cause red shift of the band of $2-3 \mathrm{~nm}$. Thus, the shifts of $28 \mathrm{~nm}$ (as observed for curve 4 Figure 5) and $13 \mathrm{~nm}$ (as observed for curve 3 Figure 5) could be explained with silver oxide shell formation around Ag nanoparticles. In this case the maximum of SPR band is red-shifted and decreases in intensity (that might be a reason for the difference in the calculated and experimental $\alpha$ values). In accordance with our previous calculations [27] the observed shift of $\lambda_{\max }$ from $390 \mathrm{~nm}$ to $403 \mathrm{~nm}$ corresponds to $r / R=0.97$ (where $r$ is the radius of $\mathrm{Ag}$ core and $R$ is the radius of whole nanoparticle). From this ratio the value of shell radius can be easily estimated, for instance nanoparticles with $R=10 \mathrm{~nm}$ have the radius of $\mathrm{Ag}_{2} \mathrm{O}$ shell equal to $0.3 \mathrm{~nm}$. For the spectrum with $\lambda_{\max }=418 \mathrm{~nm}$ the calculated $r / R$ value is smaller -0.96 and oxide shell is slightly thicker. One can see that oxide shell is rather thin, but it prevents $\mathrm{Ag}$ particle from further oxidation and agglomeration, which follows from the fact that no significant changes are observed in the colloidal solution spectra during a week. In comparison, the not coated with oxide shell, 
Ag particles quickly oxidize (during 0.5 hour) after exposure to air [7].

\section{CONCLUSIONS}

BP-modified silica films and powders have been found to be effective catalysts and sensitizers in the photoreduction of silver ions in the alcohol-water solution under irradiation with UV light at $253.7 \mathrm{~nm}$ and $365 \mathrm{~nm}$. In presence of IPA and colloidal silica, Ludox acting as a stabilizer, Ag nanoparticles have been formed. The mechanism of sensitized photoreduction of $\mathrm{Ag}^{+}$in presence of $\mathrm{SiO}_{2}$-BP films and powders, includes the $\mathrm{BP}$ anion-radical formation, which acts as a reducing agent. The formation of Ag nanoparticles passes through the stages of nucleation, aggregation and adsorption on colloid silica Ludox particles. After prolonged irradiation the nanoparticles ablation has been observed. The theoretical model suggested the silver particles stabilization in the transparent colloid silica surrounding, due to the formation of thin silver oxide shell.

\section{ACKNOWLEDGMENTS}

The authors wish to thank Professor M. A. El-Sayed for fruitful discussions and for the help and kindness at the performance of this work.

\section{REFERENCES}

[1] P. Mazzoldi, G. W. Arnold, G. Battaglin, F. Gorella, and R. F. Haglund, J. Nonlin. Opt. Phys. Mat. 5 (1996), 285.

[2] P. V. Kamat, J. Phys. Chem. B. 106 (2002), 7729.

[3] C. F. Bohren and D. R. Huffman, Absorption and Scattering of Light by Small Particles, Wiley, New York, 1983.

[4] M. Mostafavi, G. R. Dey, L. Francois, and J. Belloni, J. Phys. Chem. A. 106 (2002), 10184.

[5] P. V. Kamat, M. Flumiani, and G. V. Hartland, J. Phys. Chem. B. 102 (1998), 3123.

[6] M. Epifani, C. Giannini, L. Tapfer, and L. Vasanelli, J. Am. Ceram. Soc. 83 (2000), 2385.

[7] A. Henglein, Chem. Mater. 10 (1998), 444.

[8] N. Kometani, H. Doi, K. Asami, and Y. Yonezawa, Phys. Chem. Chem. Phys. 101 (2002), 5142.
[9] Y. Yonezawa, T. Sato, M. Ohno, and H. Hada, J. Chem. Soc., Faraday Trans. 83 (1987), 1559.

[10] J. C. Sciano, J. Photochem. 2 (1973), 81.

[11] H. A. Schwarz and R. W. Dodson, J. Phys. Chem. 93 (1989), 409.

[12] J. K. Thomas, Photochem. Photobiol. Sci. 3 (2004), 484.

[13] S. Eustis, G. Krylova, A. Eremenko, N. Smirnova, A. W. Schill, and M. El-Sayed, Photochem. Photobiol. Sci. 4 (2005), 154.

[14] A. Eremenko, N. Smirnova, L. Spanhel, O. Rusina, L. Linnik, T. B. Eremenko, and K. Rechthaler, J. Molec. Struct. 553 (2000), 1.

[15] T. Yamada, K. Asai, A. Endo, H. S. Zhou, and I. Honma, J. Mater. Sci. Lett. 19 (2000), 2167.

[16] N. Smirnova, A. Eremenko, O. Rusina, W. Hopp, and L. Spanhel, J. Sol-Gel Sci. Technol. 21 (2001), 109.

[17] C. G. Hatchard and C. A. Parker, Proc. R. Soc. London, Ser. A 235 (1956), 518.

[18] S. J. Gregg and K. S. W. Sing, Adsorption, Surface Area and Porosity, Academic Press, London, 1982.

[19] G. V. Lisichkin, A. Y. Fadeev, A. A. Serdan, P. N. Nesterenko, P. G. Mingalyov, and D. B. Furman, Chemistry of Surface Grafted Compounds, Fizmatlit, Moscow, 2003.

[20] P. Yankov, Z. Nickolov, and V. Zhelyaskov, J. Photochem. Photobiol. 47 (1988), 155.

[21] Y. Lin and D. Jonach, J. Phys. Chem. 97 (1993), 295.

[22] G. Mie, Ann. Phys. 25 (1908), 377.

[23] G. Krylova, N. Smirnova, A. Eremenko, S. Eustis, W. Huang, and M. A. El-Sayed, J. Photochem. Photobiol. A: Chem., submitted for publication.

[24] J. L. Marignier and B. Hickel, J. Phys. Chem. 88 (1984), 5375.

[25] A. Henglein, J. Phys. Chem. 97 (1993), 5457.

[26] F. Umland, A. Janssen, D. Thierig, and G. Wuensch, Theorie und Praktische Anwendung von Komplexbildnern, Ed. Akademische Verlagsgesellschaft, Frankfurt amMain, 1971.

[27] L. G. Grechko, A. M. Eremenko, L. B. Lerman, G. V. Krylova, N. P. Smirnova, and N. G. Shkoda, Proceedings of Kyiv University, Series: Physics \& Mathematics 4 (2004), 450. 


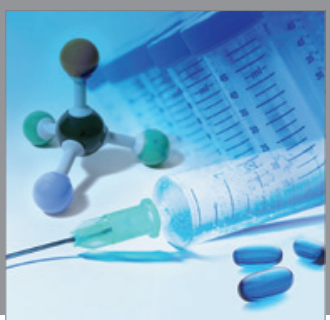

International Journal of

Medicinal Chemistry

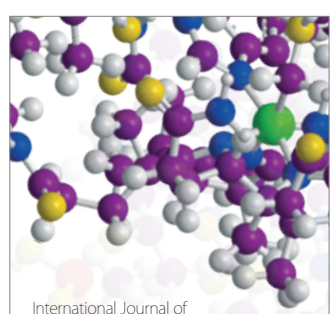

Carbohydrate Chemistry

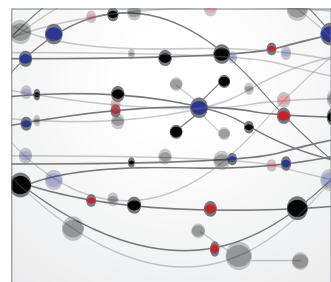

The Scientific World Journal
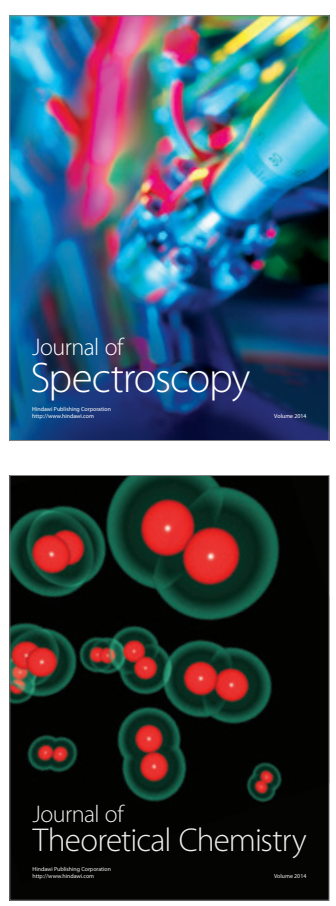
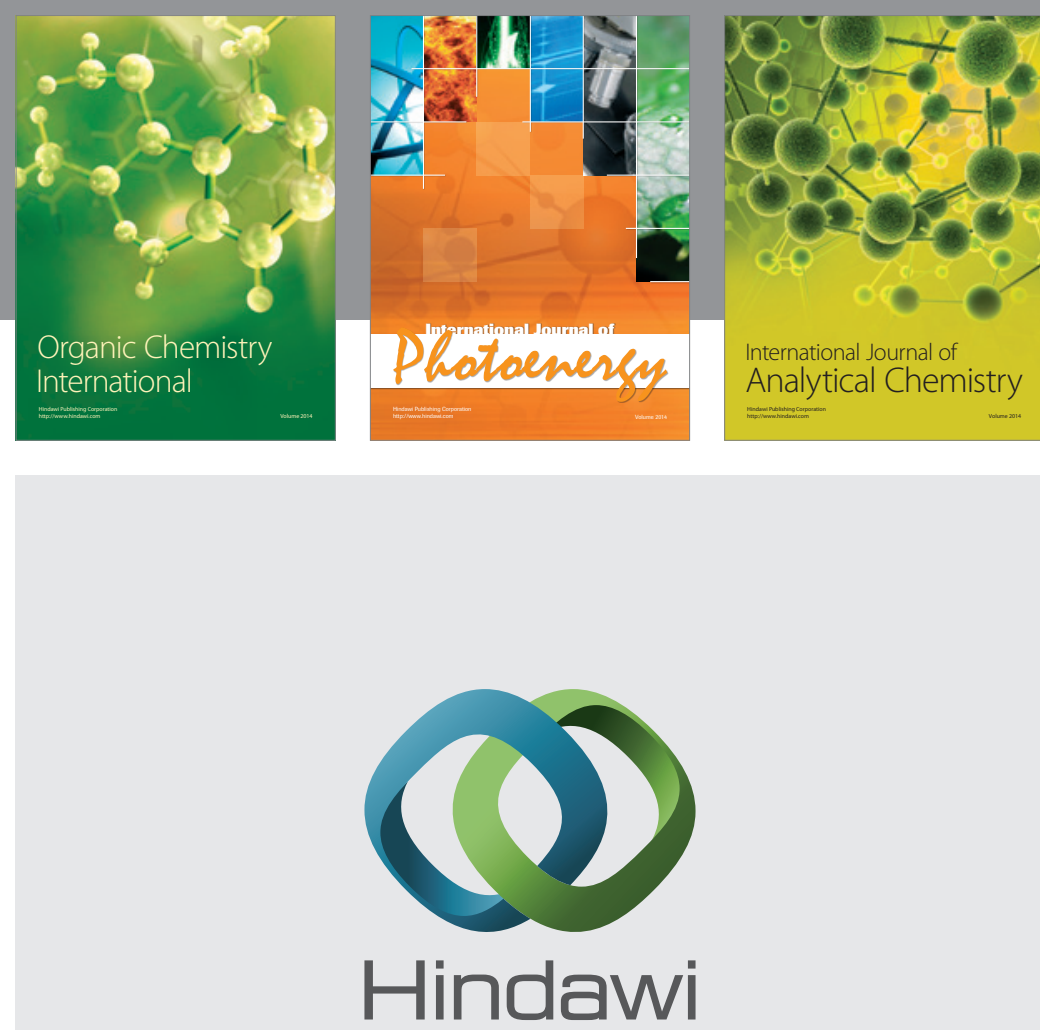

Submit your manuscripts at

http://www.hindawi.com
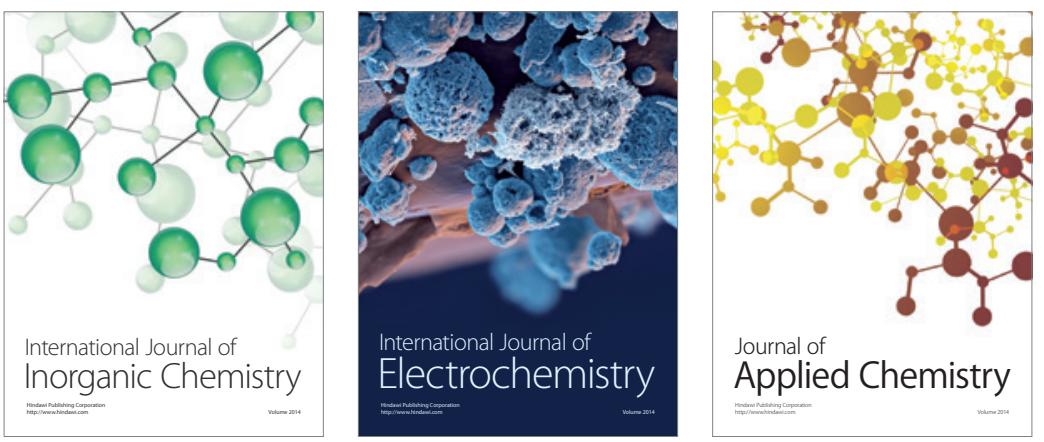

Journal of

Applied Chemistry
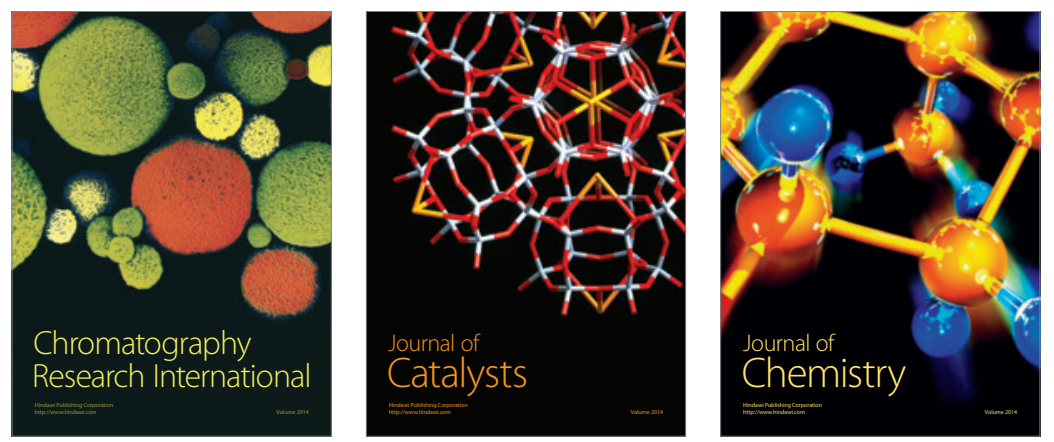
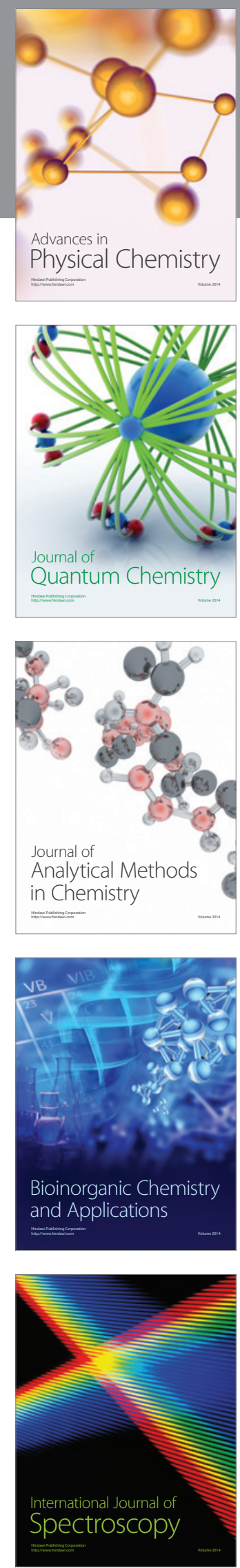